\title{
ENDOSCOPIA DEL SUEÑO MEDIANTE SEDACIÓN INDUCIDA POR FÁRMACOS O SOMNOSCOPIA
}

\section{Drug inducted sleep endoscopy or somnoscopy}

Pablo SANTOS-GORJÓN'; Juan Luis SÁNCHEZ-JARA-SÁNCHEZ'; Gonzalo MARTÍN-HERNÁNDEZ1; Ana Cristina MORALES-MARTÍN ${ }^{2}$; Jean Franco INTRAPRENDENTE-MARTINI ${ }^{1}$; Rosalyn CHAVES-ARAÚJO' ${ }^{1}$; Angélica LEÓN-RAMOS ${ }^{1}$; Erwin Alejandro RACINES-ÁLAVA ${ }^{1}$

Complejo Asistencial de Ávila. ${ }^{1}$ Servicio de Otorrinolaringología. ${ }^{2}$ Servicio de Anestesiología. Ávila. España

Correspondencia: pablosantosgor@gmail.com

Fecha de recepción: 12 de enero de 2020

Fecha de aceptación: 17 de febrero de 2020

Fecha de publicación: 1 de marzo de 2020

Fecha de publicación del fascículo: 1 de diciembre de 2020

Conflicto de intereses: Los autores declaran no tener conflictos de intereses

Imágenes: Los autores declaran haber obtenido las imágenes con el permiso de los pacientes

Política de derechos y autoarchivo: se permite el autoarchivo de la versión post-print (SHERPA/RoMEO)

Licencia CC BY-NC-ND. Licencia Creative Commons Atribución-NoComercial-SinDerivar 4.0 Internacional

Universidad de Salamanca. Su comercialización está sujeta al permiso del editor

RESUMEN: Introducción y objetivos: La somnoscopia es una herramienta diagnóstica para valorar las posibles obstrucciones de la vía aérea superior responsables del síndrome de apnea-hipopnea del sueño (SAHS). Nuestro objetivo de es mostrar nuestra experiencia con somnoscopia o DISE (endoscopia mediante sedación inducida por fármacos) en candidatos a cirugía por una patología obstructiva de vía aerodigestiva superior. Material y métodos Presentamos un estudio retrospectivo de 17 pacientes de los que se excluyen finalmente 2. La edad de los pacientes varió de 34 a 72 años. Se practica polisomnografía previa y el IAH medio fue 7,32 $\pm 8,71$. Discusión y conclusión: La DISE es una técnica segura, reproducible y aplicable en cualquier servicio de ORL. Así mismo, permite individualizar el tratamiento quirúrgico y probablemente evitar cirugías innecesarias.

PALABRAS CLAVE: Síndrome de apnea obstructiva del sueño; somnoscopia; DISE. 
SUMMARY: Introduction and objectives: Somnoscopy can Valore the possible obstruction in superior aerodigestive tract, that lead to obstructive sleep apnea syndrome (OSAS). Our objective is showing our experience using the somnoscopy or DISE (drug induced sleep endoscopy). Material and methods: We present a retrospective study of 17 patients. Finally, two patients were excluded. The patients were 34-72 years old. A Polysomnography was done, and hypopneaapnea index was 7,32 $\pm 8,71$. Discussion and conclusions: This a secure technique and can be reproduced in all ENT services. DISE less us individualize chirurgical treatment and avoid us unnecessary patients' operations.

KEYWORDS: Obstructive sleep apnea syndrome; somnoscopy; DISE.

\section{INTRODUCCIÓN}

El SAHS es un problema cada vez más prevalente en nuestro ámbito [1,2]. Muchas veces está asociado a nuestro estilo de vida sedentario, envejecimiento de los pacientes que atendemos y su pluripatología acompañante [3,4]. La endoscopia mediante sedación inducida por fármacos, somnoscopia o DISE (drug induced sleep endoscopy) es una prueba de carácter diagnóstico. Complementa a la videopolisomnografía nocturna para un diagnóstico integral de los problemas obstructivos relacionados con el sueño [5]. Se usa principalmente en el síndrome de apnea del sueño (SOAS), pero también puede tener su utilidad en ronquido simple [6].

Nuestra experiencia inicial con esta exploración ha sido muy satisfactoria. La usamos de forma complementaria en algunas de nuestras cirugías programadas. En un principio se planteó la posibilidad en nuestro servicio de buscar una alternativa sólo para pacientes con diagnóstico de síndrome obstructivo con apnea del sueño (SOAS) mediante estudio con polisomnografía patológica y en aquellos pacientes que no tolerasen el dispositivo de presión positiva continua en la vía aérea (CPAP, Continuous Positive Airway Pressure). Este dispositivo continúa siendo hoy día el tratamiento de elección en pacientes con apnea moderada a severa sintomático.

Así, en pacientes intolerantes a CPAP por el motivo que fuera se puede plantear la opción quirúrgica si presentan una patología orgánica obstructiva de vía alta. Ya que dicho paciente va a ser sometido a una anestesia general, al incluirlo en lista de espera quirúrgica, incluimos en el procedimiento quirúrgico que el paciente precise la DISE.

El objetivo de este estudio es mostrar nuestra experiencia inicial con dicha técnica y valorar si realmente nos aporta información para ayudar en nuestra práctica diaria.

\section{MATERIAL Y MÉTODO}

Estudio retrospectivo de 17 pacientes consecutivos a los que se realizó DISE en el último año en nuestro servicio. En este estudio se incluyeron 15 pacientes en los que se realizó diagnóstico topográfico de su obstrucción ventilatoria mediante la somnoscopia. Uno de los dos pacientes presentaba sólo roncopatía porque su polisomnografía no cumplía criterios patológicos. El otro paciente no disponía de estudio documentado de SOAS como tal.

Todos los pacientes fueron diagnosticados por el servicio de neumología (quien realiza los estudios del sueño en nuestro centro). La edad de los pacientes varió de 34 a 72 años. Se practicó polisomnografía previa. El IAH (índice de apneas-hipopneas) medio fue $7,32 \pm 8,71$. En esta serie hay el doble de varones que mujeres (10/5). Varios miembros del servicio realizaron una formación específica teórica y práctica. Se celebró una sesión conjunta con anestesiología para realizar un protocolo y comenzar a realizar DISE ya que precisamos monitorización de nivel de profundidad anestésica y monitorización 
neurológica mediante BIS (biespectral index). La sedación se realiza mediante bomba de propofol si es posible (si no está disponible se realiza mediante bolos de Propofol) con el objetivo de mantener un DISE de entre 50 y 70 . El colapso multinivel se etiqueta mediante la escala VOTE (velo de paladar blando, paredes laterales velofaríngeas, paredes laterales de orofaringe incluidas amígdalas palatinas, base de lengua y Epiglotis). La escala VOTE se recoge en el protocolo quirúrgico informatizado mediante un cómodo sistema de marcado de aspas que nos adapta nuestro servicio de Informática para su digitalización inmediata en la historia clínica electrónica.

Tabla 1. Protocolo quirúrgico. Escala VOTE.

\begin{tabular}{|c|l|l|}
\hline & $\begin{array}{l}\text { Posición paciente en } \\
\text { decúbito supino }\end{array}$ & Tipo de colapso \\
\hline V & Velo del paladar & $\begin{array}{l}\text { Lateral Anteroposterior } \\
\text { Concéntrico }\end{array}$ \\
\hline O & $\begin{array}{l}\text { Paredes laterales de } \\
\text { orofaringe incluidas } \\
\text { amígdalas palatinas }\end{array}$ & $\begin{array}{l}\text { Lateral Anteroposterior } \\
\text { Concéntrico }\end{array}$ \\
\hline T & Lengua & $\begin{array}{l}\text { Lateral Anteroposterior } \\
\text { Concéntrico }\end{array}$ \\
\hline E & Epiglotis & $\begin{array}{l}\text { Lateral Anteroposterior } \\
\text { Concéntrico }\end{array}$ \\
\hline & Colapso multinivel & Si/No \\
\hline
\end{tabular}

Tabla 2. Maniobras durante la somnoscopia.

\begin{tabular}{|l|l|}
\hline Tipo de maniobra & Corrección \\
\hline Chin Lift & $\mathrm{Si} /$ no \\
\hline Oclusión de labios & $\mathrm{Si} /$ no \\
\hline Avance mandibular & $\mathrm{Si} /$ no \\
\hline Rotación cefálica derecha & $\mathrm{Si} /$ no \\
\hline Rotación cefálica izquierda & $\mathrm{Si} /$ no \\
\hline
\end{tabular}

En la Tabla 1 se recoge la escala VOTE, la Tabla 2 las maniobras durante la somnoscopia. La Figura 1 muestra el protocolo quirúrgico del sistema informático y la Figura 2 las maniobras realizadas y observaciones subjetivas de los exploradores.

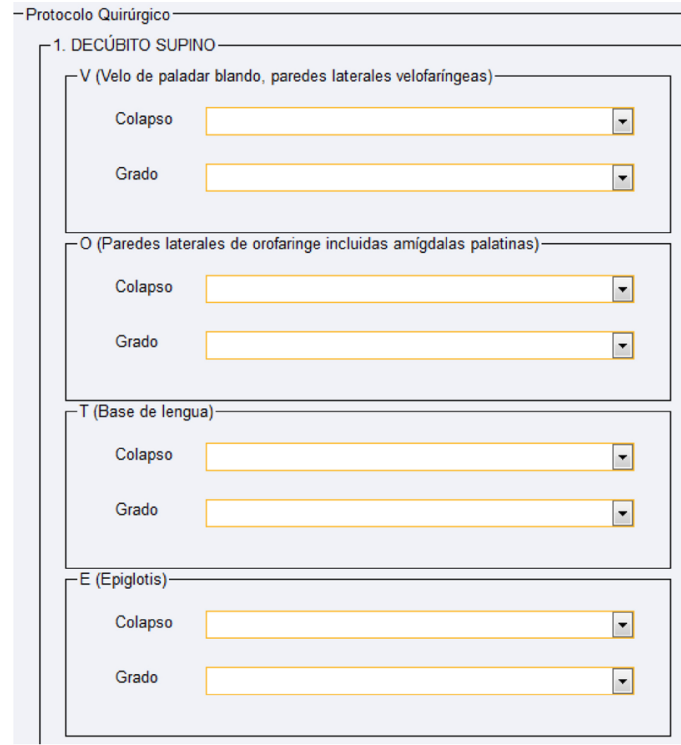

Figura 1. Protocolo quirúrgico tipo en el sistema informático.

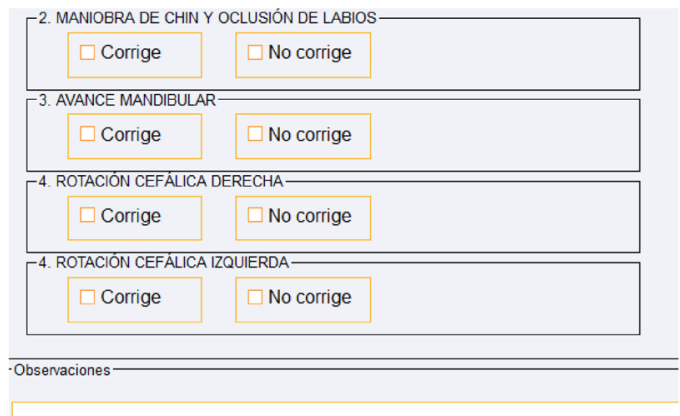

Figura 2. Protocolo quirúrgico. Maniobras realizadas y observaciones subjetivas de los exploradores.

\section{RESULTADOS}

Con avance mandibular en 7 de los pacientes (50\%) mejoró el grado de apnea. Se observó un colapso circular de la vía aérea en un $40 \%$ de los 
pacientes. En otro $40 \%$ el colapso predominante fue anteroposterior. Además, 3 pacientes presentaban un problema bajo obstructivo con colapso epiglótico sobre vía aérea.

En otros 6 casos (40\%) se vio implicada la base de la lengua.

En todos los casos, tras la DISE se procedió a intubar al paciente y realizar la cirugía prevista a continuación. Se realizó un protocolo quirúrgico independiente de la DISE en la historia electrónica y otro de la cirugía. El protocolo se realizó de forma consensuada con los dos cirujanos presentes durante la exploración.

Los resultados se resumen en la Tabla 3.

Tabla 3. Resultados. Escala VOTE.

\begin{tabular}{|c|c|c|c|}
\hline Nivel & Vibración & Colapso & Dirección \\
\hline Velo & $\begin{array}{l}80 \% \\
(12 \\
\text { pacientes })\end{array}$ & $\begin{array}{l}80 \% \\
(12 \\
\text { pacientes })\end{array}$ & $\begin{array}{l}40 \% \text { ( } 6 \text { pacientes) } \\
\text { anteroposterior } \\
40 \% \text { ( } 6 \text { pacientes) } \\
\text { concéntrico }\end{array}$ \\
\hline $\begin{array}{l}\text { Orofaringe } \\
\text { (amígdalas) }\end{array}$ & $\begin{array}{l}15 \% \\
(2 \\
\text { pacientes })\end{array}$ & $\begin{array}{l}20 \% \\
(3 \\
\text { pacientes })\end{array}$ & $\begin{array}{l}20 \% \text { ( } 3 \text { pacientes) } \\
\text { lateral }\end{array}$ \\
\hline $\begin{array}{l}\text { Base de } \\
\text { lengua }\end{array}$ & $\begin{array}{l}10 \% \\
(2 \\
\text { pacientes })\end{array}$ & $\begin{array}{l}40 \% \\
(6 \\
\text { pacientes })\end{array}$ & $\begin{array}{l}20 \% \text { ( } 3 \text { pacientes) } \\
\text { anteroposterior } \\
5 \%(2) \text { lateral }\end{array}$ \\
\hline Epiglotis & $\begin{array}{l}15 \% \\
(2 \\
\text { pacientes })\end{array}$ & $\begin{array}{l}20 \% \\
(3 \\
\text { pacientes })\end{array}$ & $\begin{array}{l}20 \% \text { ( } 3 \text { pacientes) } \\
\text { anteroposterior } \\
5 \%(1) \text { lateral }\end{array}$ \\
\hline Multinivel & $60 \%$ & $60 \%$ & \\
\hline
\end{tabular}

\section{DISCUSIÓN}

La prueba consiste en la inducción de un sueño corto (menor de 20 minutos) mediante fármacos que permite asimilar los resultados obtenidos de manera fiable al sueño natural [7]. En esta prueba no se pretende realizar un estudio de parámetros relativos a la gravedad del ronquido, la presencia o no de apneas o a la calidad del sueño. Pretendemos encontrar el puntos o los puntos de bloqueo de la vía respiratoria responsables de la apnea o el ronquido [8]. Se ha comprobado que muchos pacientes presentan patología multinivel. De este modo si mejoramos su función nasal, pero persiste una obstrucción más baja persistirá en mayor o menor grado un SAOS [9]. La somnoscopia es una prueba segura y carente prácticamente de efectos secundarios, pero debe realizarse en condiciones de seguridad y con las constantes del paciente monitorizadas [10]. En nuestro medio lo realizamos en el quirófano y con un anestesista siempre. El ORL explora mediante una fibroscopio habitual de $4 \mathrm{~mm}$ la vía aérea e identifica de manera directa los puntos de vibración (ronquido) y los puntos de obstrucción (apnea) evaluando la posibilidad de mejora de estos mediante las distintas modalidades terapéuticas (medidas posturales e higiénicas, CPAP, avance mandibular o similitud con la cirugía prevista de realizar). Así si introducimos un tubo tipo Rush de intubación en la faringe podremos por ejemplo simular la cirugía de una faringoplastia y ver si mejoraría el grado obstructivo antes de realizarla. Es muy importante la concienciación anestesiología y explicarle lo que pretendemos y no pretendemos hacer con esta exploración. Esto, si tenemos en cuenta que se trata de pacientes pluripatológicos, que en muchos casos son intubación difícil y con un elevado riesgo anestésico no siempre es fácil. Además, cada paciente tiene un metabolismo anestésico diferente y es difícil mantener un BIS por debajo de 70 y que el paciente permita la manipulación endoscópica de la vía aérea durante toda la prueba de somnoscopia [11]. Es recomendable el uso de una torre endoscópica que favorezca que el cirujano principal y el ayudante tengan visión de la fibroscopia durante toda la exploración. Se consensúa la puntuación de la prueba entre los dos ORL presentes en el quirófano. La introducción de la DISE en nuestro servicio permite mejorar las indicaciones quirúrgicas para la cirugía del ronquido, o incluso contraindicarla. La puntuación 
que el cirujano obtenga durante la prueba en los diferentes niveles de la VADS sirve para proponer la cirugía o intervenciones sobre la vía aérea [12]. Es frecuente que se objetive que con avance mandibular en 7 de nuestros pacientes (50\%) mejora el grado de apnea. Ahora bien, el Dispositivo de Avance Mandibular (DAM) es un dispositivo relativamente caro y que el paciente debe solicitar a su odontólogo de referencia y realizar a medida. Debido a este inconveniente sólo la mitad de los pacientes realizaron dicha adaptación finalmente. Otros hallazgos fueron un colapso circular de la vía aérea en un $40 \%$ de los pacientes explorados. Este hallazgo es el más difícil de solucionar de forma quirúrgica. En otro $40 \%$ de los pacientes el colapso predominante es anteroposterior y se beneficiarían de una faringoplastia. Además, en 3 pacientes presentaban además un problema bajo obstructivo con colapso epiglótico sobre vía aérea. En estos pacientes podría plantearse una resección epiglótica. En otros 6 pacientes se vio implicada la base de la lengua. De momento no hemos realizado ninguna resección basilingual. De todas maneras, hemos observado que hay una diferencia entre las indicaciones quirúrgicas basadas en la exploración clínica y la que finalmente resultó tras el DISE en más casi la mitad de nuestros pacientes. Esto es congruente con la literatura revisada. Hay que tener en cuenta que estas cirugías normalmente implican un postoperatorio tórpido, doloroso y que debemos exponer al paciente el objetivo de la cirugía en caso de realizarla. En nuestro medio la espera media para realizar un estudio del sueño es bastante larga. Tras realizar la somnoscopia y la cirugía pertinente solemos precisar otro estudio del sueño para comparar con el previo. Puesto que estamos hablando de pacientes seleccionados y estimamos que pueden precisarse en nuestro medio menos de 30 estudios del sueño más anuales y creemos que sería asumible en nuestro centro dicho número de pacientes. Se puede plantear realizar DISE en pacientes de forma más rutinaria si disponemos de una sala de exploración o endoscopia fuera del quirófano. En nuestro centro debido a la escasez tanto de quirófanos como de anestesistas de momento no es podemos plantearlo. Puede pensarse que debido a la realización de la DISE se puede alargar mucho el tiempo anestésico. Debemos explicar adecuadamente el objetivo de esta exploración, y que en muchas ocasiones vamos a evitar cirugías invasivas y postoperatorios tórpidos y dolorosos si comprobamos que no van a mejorar el ronquido ni la apnea en vivo.

\section{CONCLUSIONES}

La DISE es una técnica segura, reproducible y aplicable en cualquier servicio de ORL. Podría plantearse su uso de forma complementaria a cirugías programadas para no ocupar quirófanos o salas de exploración en las que de todas maneras precisaremos la presencia de un anestesista.

La DISE permite individualizar el tratamiento quirúrgico y probablemente evitar cirugías innecesarias.

\section{BIBLIOGRAFÍA}

1. Rich J, Raviv A, Raviv N, Brietzke S.E. Allcause mortality and obstructive sleep apnea severity revisited. Otolaryngol Head Neck Surg. 2012;147:58387.

2. Pieters T, Collard P, Aubert G, Dury M, Delguste $\mathrm{M}$, Rodenstein DO. Acceptance and long-term compliance with nCPAP in patients with obstructive sleep apnoea syndrome. Eur Respir Physiol. 2016;15:939-44.

3. Sundaram S, Bridgman SA, Lim J, Lasserson TJ. Surgery for obstructive sleep apnoea. Cochrane Database Syst Rev. 2005;19:4. https://www.ncbi. nlm.nih.gov/pubmed/16235277

4. Sher AE, Schechtman KB, Piccirillo JF. The efficacy of surgical modifications of the upper airway in adults with obstructive sleep apnea syndrome. Sleep. 1996;15:156-7.

5. Borowiecki B, Pollak CP, Weitzman ED, Rakoff S, Imperato J. Fibro-optic study of pharyngeal airway during sleep in patients with hypersomnia 
obstructive sleep-apnea syndrome. Laryngoscope. 2008;88:1310-13.

6. Croft CB, Pringle M. Sleep nasendoscopy: A technique of assessment in snoring and obstructive sleep apnoea. Clin Otolaryngol Allied Sci. 1991;16: 504-9.

7. Rabelo FAW, Braga A, Küpper DS, De Oliveira JAA, Lopes FM, de Lima Mattos PLV. Propofolinduced sleep: polysomnographic evaluation of patients with obstructive sleep apnea and controls. Otolaryngol Head Neck Surg. 2010;142: 218-24.

8. Hillman DR, Walsh JH, Maddison KJ, Platt PR, Kirkness JP, Noffsinger WJ. Evolution of changes in upper airway collapsibility during slow induction of anesthesia with propofol. Anesthesiology. 2009;111: 63-71.
9. Babar-Craig H, Rajani NK, Bailey P, Kotecha BT. Validationof sleep nasendoscopy for assessment of snoring with bispectral index monitoring. Eur Arch Otorhinolaryngol.2012; 269: 1277-9.

10. Abdullah VJ, Lee DLY, Ha SCN, van Hasselt CA. Sleep endoscopy with midazolam: Sedation level evaluation with bispectral analysis. Otolaryngol Head Neck Surg. 2013;148: 331-7.

11. Gillespie MB, Reddy RP, White DR, Discolo CM, Overdyk FJ, Nguyen SA. A trial of drug-induced sleep endoscopy in the surgical management of sleep-disordered breathing. Laryngoscope. 2013;123: 277-82.

12. El Badawey MR, McKee G, Heggie N, Marshall $\mathrm{H}$, Wilson JA. Predictive value of sleep nasendoscopy in the management of habitual snorers. Ann Otol Rhinol Laryngol. 2003;112: 40-4. 